\title{
Lagrangian Trajectory Simulation of Rotating Regular Shaped Ice Particles
}

\author{
Markus Widhalm \\ DLR Braunschweig \\ Lilienthalplatz 7 \\ 38108 Braunschweig \\ markus.widhalm@dlr.de
}

\begin{abstract}
This paper focuses on the numerical simulation of the motion of regular shaped ice particles under the forces and torques generated by aerodynamic loading. Ice particles can occur during landing and take-off of aircraft at ground level up to the lower bound of the stratosphere at cruising altitude. It may be expected that the particle Reynolds number is high because the flow around the aircraft is in certain regions characterized by strong acceleration and deceleration of the flow. In combination with this flow pattern, the rotation of particles becomes important. Applicable translational and rotational equations of motion combined with a drag correlation taking into account rotation will be derived for a Lagrangian type particle tracking. Orientation is described with quaternions to prevent the singularities associated with the description by Euler angles. The influence of regular shaped particles on collection efficiencies is investigated. Test cases are the flow past a cylinder, a NACA0012 airfoil and a NHLP L1/T2 three element airfoil. Due to the increased computational effort compared to the purely translational approach, observed trajectory simulation times are reported.
\end{abstract}

\section{Nomenclature}

\section{Variables}

$A=$ surface or projected area, $m^{2}$

$C_{D}=$ aerodynamic drag coefficient

$C_{L}=$ aerodynamic lift coefficient

$d \quad=\quad$ diameter, $m$

$\vec{F} \quad=$ force vector, $N$

$\mathrm{I}=$ moment of inertia tensor, $\mathrm{kg} \mathrm{m}^{2}$

$L \quad=\quad$ length of particle, $m$

$m \quad=$ mass, $\mathrm{kg}$

$\mathrm{Ma}_{\infty}=$ freestream Mach number

$R \quad=$ rotation matrix

Re $\quad=$ Reynolds number

$\mathrm{Sp} \quad=$ Spin number

$\vec{T} \quad=$ torque vector, $\mathrm{Nm}$

$t \quad=$ physical time, $s$

$\vec{x} \quad=$ position vector, components $x, y$ and $z, m$

\section{Greek letters}

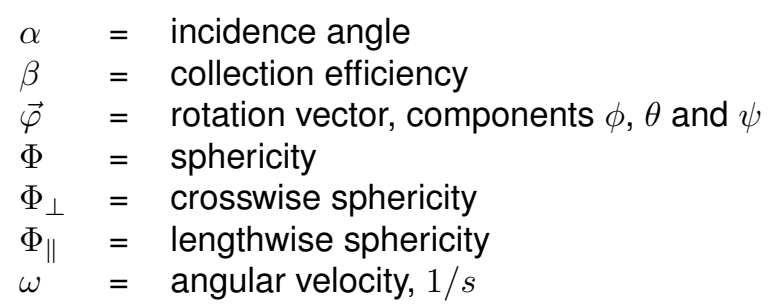

\section{Subscripts}

$\begin{aligned} p & =\text { particle state } \\ f & =\text { surrounding fluid state } \\ r & =\text { resistance } \\ R & =\text { rotation } \\ c . p . & =\text { center of pressure } \\ c . g . & =\text { center of gravity } \\ \infty & =\text { freestream state } \\ \text { ref } & =\text { reference state } \\ \text { rel } & =\text { relative state }\end{aligned}$

\section{Introduction}

Ice crystals may be found at the upper boundary of the troposphere where strong winds exist and jet aircraft fly at cruising speed in the transonic flow regime and high flow Reynolds number. Ice crystals found in this part of the atmosphere deviate considerably in shape from spheres and may appear as thin needles up to disc-shaped structures. As the particle size grows from a few microns to several hundreds of microns a high particle Reynolds number is to be expected as well. These conditions will most likely cause an arbitrary rotation and orientation of the particle around its principal axes.

However, as drag is the most important force contribution, early investigations focused on the drag coefficients of spheres moving through a fluid with relatively low flow and particle Reynolds numbers. By a combination of theoretical work and extensive experimental testing a huge amount of data was collected. It resulted in an empirical correlation of the drag coefficient, dependent on the particle Reynolds number. Extension of these correlations to 
irregular shaped particles happened in small steps. Initially, a limited number of regular shaped particles was considered by determining the settling behavior and keeping track of the free-falling velocity and drag. Many of these investigations have been carried out with prisms, cylinders, cones or plates. Again, empirical drag correlations were proposed with an additional parameter, often referred to as sphericity. Sphericity is the ratio between a certain characteristic description (e.g. the cross section) of the equivalent sphere and the non-spherical particle. By definition, sphericity does not account for the orientation of the particle in relation to the direction of fluid flow. The main outcome was an increased drag coefficient for non-spherical particles in comparison to spherical particles. For years, averaged or stochastic correlation were used to determine drag coefficients based on huge sets of experimental data for different, mostly regular shaped, particles [1, 2, 3] and have been extended to irregular shaped particles to provide a reliable drag correlation that covers as many shapes as possible. The computational effort to evaluate the drag coefficient will be comparable to spherical particles.

However, if the particle Reynolds number increases considerably and experimental investigations become difficult, considering the full particle motion including rotation may significantly enhance the trajectory simulation. One of the first investigations was done by Jeffery [4] for ellipsoids and by Cox [5] for slender bodies, both comprehensive theoretical studies. The influence of particle rotation at higher particle Reynolds numbers was recently pointed out by Qi [6] while investigating sedimentation. Huang et.al. [7] identified different modes of particle motion related to a wide range of particle Reynolds numbers for spheroidal particles in a Couette flow. Rosendahl [8] introduced a multi-parameter description of the particle rotation to improve prediction capability of numerical simulations instead of using a general single parameter like the sphericity. Comprehensive overview article on extensions to more general regular and irregular shaped particles may be found in Loth [9], Mandø and Rosendahl [10] and Kleinstreuer and Feng [11]. Experimental investigations of rotating balls in a turbulent flow were examined by Zimmermann et. al. [12].

Obviously, the general treatment of non-spherical particles needs to consider the shape and orientation dependent aerodynamic forces, which are associated with nonspherical particles. The computation of both the aerodynamic forces and torques and of the particle motion requires the tracking of the particle orientation and rotation plus the formulation of appropriate orientation dependent lift and drag correlations. If the translational and rotational equations of motion are applied, a set of ordinary differential equations emerges, which introduce external forces and torques. To simplify evaluation of forces and torques, we delimit our investigation in this paper to the treatment of regular shaped particles only. The forces and torques may then be evaluated using orientation based geometrical parameters. Certain parameters are derived from experiments to keep the computational effort within bounds.

The original DLR TAU Lagrangian-type particle tracer, as described in [13], could only consider translational motion of particles. The equations of motion are derived from Newton's second law for a point mass, but consider drag, buoyancy and gravity forces. The drag correlation implemented is based on a fit to Langmuir and Blodgett's [14] drag data for spherical water droplets in a dispersed flow. The particle tracer was mainly established to evaluate water droplet collection efficiencies for subsequent ice accretion simulations. The resulting ordinary differential equation (ODE) is solved in time with embedded Runge-Kutta methods of third or fourth order. An extension to the original implementation, solving in addition the rotational equations of motion and the equations for evaluating the orientation, is described in this paper. As irregular shapes are difficult to treat and the accurate aerodynamics around the particle surface is not resolved in our approach, restriction to regular shaped particles are necessary. We consider both discs and rods with specified aspect ratios, for which the equation of motion can be derived by basic mechanical considerations since experimental data are rare. Aerodynamic forces and corresponding torques are introduced from existing correlations which account for orientation and non-spherical parameters.

A suitable drag correlation approach was introduced by Hölzer and Sommerfeld [15], which depends on the particle Reynolds number, lengthwise and crosswise sphericity and a general description of the sphericity. Lift and resistance torque against the rotation is treated as proposed by Mandø and Rosendahl [10]. Computation of particle orientation angles and angular velocity is done in the principal axis system of the considered particle using Euler's equations for a rotational motion. Orientation is represented using quaternions. However, the quaternion representation needs to be transferred back into Euler angles to recompute projected areas and equivalent sphere parameters. The differential equations for rotational and the translational motion based on Newton's law form together a system of ordinary differential equations, which is again solved in time by embedded Runge-Kutta integrators with local error control. The demand of this solution process on computer time is outlined too, which is a limiting factor for the potential use in industrial applications, whenever orientation of particles is considered. Evaluation of collection efficiencies for selected test cases will emphasize the influence of particle rotation in comparison to averaged correlation approaches. 


\section{Governing equations of particle mo- tion}

The governing equations for the translational and rotational motion of a particle in a fluid is Newton's second law in a global frame of reference,

$$
\begin{aligned}
\vec{x}_{p}(t) & :=\left[x_{p}(t), y_{p}(t), z_{p}(t)\right]^{T}, \\
\frac{\mathrm{d} \vec{x}_{p}(t)}{\mathrm{d} t} & :=\dot{\vec{x}}_{p}(t)=\vec{U}_{p}(t), \\
m_{p} \frac{\mathrm{d} \vec{U}_{p}(t)}{\mathrm{d} t} & :=\sum \vec{F}(t),
\end{aligned}
$$

and Euler's equations of motion in a body fixed frame of reference

$$
\begin{aligned}
\vec{\varphi}_{p}(t) & :=[\phi(t), \theta(t), \psi(t)]^{T}, \\
\frac{\mathrm{d} \vec{\varphi}_{p}(t)}{\mathrm{d} t} & :=\dot{\vec{\varphi}}_{p}(t)=\vec{\omega}_{p}(t), \\
\mathrm{I}_{p} \frac{\mathrm{d} \vec{\omega}_{p}(t)}{\mathrm{d} t}+ & \vec{\omega}_{p}(t) \times\left(\mathrm{I}_{p} \vec{\omega}_{p}(t)\right):=\sum \vec{T}(t), \\
\mathrm{I}_{p} & :=\left[\begin{array}{lll}
I_{x x} & I_{x y} & I_{x z} \\
I_{y x} & I_{y y} & I_{y z} \\
I_{z x} & I_{z y} & I_{z z}
\end{array}\right]
\end{aligned}
$$

where $m_{p}$ is the particle mass and $\mathrm{I}_{p}$ is the particle's moments of inertia tensor, respectively. Both are kept constant. In the present paper, the index $p$ denotes a particle property whereas index $f$ is used for properties of the surrounding fluid. Furthermore, Figure 1 shows the body fixed coordinate system $[x, y, z]$ and the geodesic reference coordinate system $\left[x^{g}, y^{g}, z^{g}\right]$.

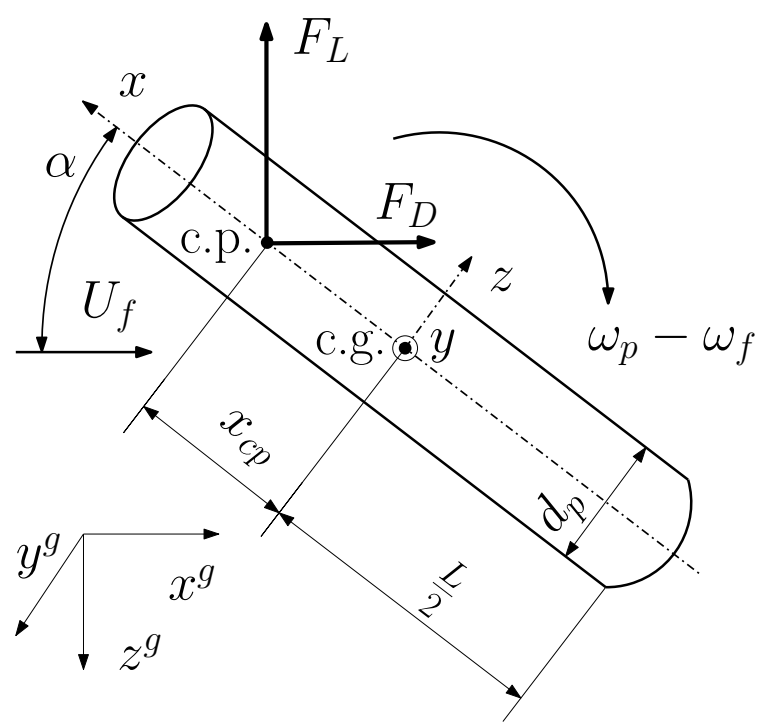

Figure 1: Aerodynamic forces acting at the center of pressure (c.p.) on a slender body and showing the relationship between geodesic $\left[x^{g}, y^{g}, z^{g}\right]$ and body fixed $[x, y, z]$ coordinate system.
It is not appropriate to solve the Eq. (5) directly with a rotation matrix based on Euler angles due to an inherent singularity, well-known as gimbal lock. It appears whenever the second rotation turns the first rotation axis parallel to the third axis of rotation, and the sequence of rotations loses one degree of freedom, [12, 16]. An alternative representation of the orientation, preventing gimbal locks, is a representation by four variables called quaternions and introduced as generalized coordinates to solve the equations of rotation. After choosing an appropriate Euler rotation sequence (3-1-3), described in [17], the quaternion parameters $q(t) \in \mathbb{R}^{4}$ and $q:=q(t)$ are defined as, [18, 17],

$$
\begin{aligned}
q & :=\left[q_{0},\left(q_{1}, q_{2}, q_{3}\right)\right]^{T}, \\
q_{0} & :=\cos \frac{\theta}{2} \cos \left(\frac{\phi+\psi}{2}\right), \\
q_{1} & :=\sin \frac{\theta}{2} \cos \left(\frac{\phi-\psi}{2}\right), \\
q_{2} & :=\sin \frac{\theta}{2} \sin \left(\frac{\phi-\psi}{2}\right), \\
q_{3} & :=\cos \frac{\theta}{2} \sin \left(\frac{\phi+\psi}{2}\right)
\end{aligned}
$$

The angular velocity transformation matrix from a bodyfixed frame of reference to a geodesic frame of reference and vice versa can be written as

$$
\begin{aligned}
& \vec{\omega}_{p}^{g}:=q \wedge \vec{\omega}_{p} \wedge \bar{q}, \\
& \vec{\omega}_{p}:=\bar{q} \wedge \vec{\omega}_{p}^{g} \wedge q
\end{aligned}
$$

where $\bar{q}$ denotes the conjugate quaternion, $q \wedge \bar{q} \equiv 1$ if the quaternion is normalized to 1 and $\wedge$ is the Grassmann product. Defining the product of Eq. (13) is a matter of convention, since it changes the effect of rotation direction. Eq. (13) results in a clockwise rotation.

Switching from quaternion to a matrix notation leads to the following alternative formulation

$$
\vec{\omega}_{p}^{g}:=R(q) \vec{\omega}, \quad \vec{\omega}_{p}:=R^{T}(q) \vec{\omega}_{p}^{g} .
$$

Since for a unit quaternion the following equivalence holds $R^{-1}(q) \equiv R^{T}(q) \equiv R(q)$, the orthogonal rotation matrix $R(q)$ is then defined by

$$
\begin{aligned}
& R(q)=\left[\begin{array}{lll}
r_{11} & r_{12} & r_{13} \\
r_{21} & r_{22} & r_{23} \\
r_{31} & r_{32} & r_{33}
\end{array}\right]= \\
& =\left[\begin{array}{ccc}
\frac{1}{2}-q_{2}^{2}-q_{3}^{2} & q_{3} q_{0}-q_{2} q_{1} & q_{1} q_{3}+q_{2} q_{0} \\
-\left(q_{2} q_{1}+q_{3} q_{0}\right) & \frac{1}{2}-q_{1}^{2}-q_{3}^{2} & q_{0} q_{1}-q_{2} q_{3} \\
q_{1} q_{3}-q_{2} q_{0} & -\left(q_{2} q_{3}+q_{1} q_{0}\right) & \frac{1}{2}-q_{1}^{2}-q_{2}^{2}
\end{array}\right]
\end{aligned}
$$

The implementation of $R(q)$ is introduced as $2 R(q)$ to save the multiplication of Eq. 17) by 0.5 . The equation of state 
variables for each particle satisfies the equation

$$
\begin{aligned}
\dot{q} & =\frac{1}{2}\left(\vec{\omega}_{p}^{g} \wedge q\right)=\frac{1}{2}\left(q \wedge \vec{\omega}_{p}\right)=\frac{1}{2} Q(q)\left[\begin{array}{c}
0 \\
\vec{\omega}_{p}
\end{array}\right] \\
Q(q) & =\left[\begin{array}{cccc}
q_{0} & -q_{1} & -q_{2} & -q_{3} \\
q_{1} & q_{0} & q_{3} & -q_{2} \\
q_{2} & -q_{3} & q_{0} & q_{1} \\
q_{3} & q_{2} & -q_{1} & q_{0}
\end{array}\right]
\end{aligned}
$$

An equation defining the second derivative of $q, \ddot{q}:=$ $\ddot{q}\left(q(t), \dot{\omega}_{p}(t)\right)$, is obtained by differentiating Eq. 177). This results in a second-order ODE for $q$,

$$
\begin{aligned}
& \ddot{q}=\frac{1}{2}\left(\dot{q} \wedge \vec{\omega}_{p}+q \wedge \dot{\vec{\omega}}_{p}\right), \quad \vec{\omega}_{p}=2(\bar{q} \wedge \dot{q}) \\
& \ddot{q}=\frac{1}{2}\left[\begin{array}{c}
|\dot{q}| \\
0
\end{array}\right]+\frac{1}{2}\left(q \wedge \dot{\vec{\omega}}_{p}\right)=\frac{1}{2} Q(q)\left[\begin{array}{c}
0 \\
\dot{\vec{\omega}}_{p}
\end{array}\right]
\end{aligned}
$$

Transformation from quaternions back to Euler angles is provided with the rotation matrix in Euler angles

$$
\begin{aligned}
R_{313}\left(\vec{\varphi}_{p}\right) & =\left[\begin{array}{ccc}
c_{\phi} c_{\psi}-s_{\phi} c_{\theta} s_{\psi} & c_{\psi} s_{\phi}+c_{\phi} c_{\theta} s_{\psi} & s_{\psi} s_{\theta} \\
-s_{\phi} c_{\psi}-s_{\phi} c_{\theta} c_{\psi} & -s_{\phi} s_{\psi}+c_{\phi} c_{\theta} c_{\psi} & c_{\psi} s_{\theta} \\
s_{\theta} s_{\phi} & -s_{\theta} c_{\phi} & c_{\theta}
\end{array}\right] \\
s_{\varphi} & :=\sin (\varphi), \quad c_{\varphi}:=\cos (\varphi) .
\end{aligned}
$$

Equivalent to matrix $R(q)$, Eq $(16)$, the Euler angles are defined by

$$
\vec{\varphi}_{p}(R(q)):=\left[\begin{array}{c}
\arctan 2\left(r_{31},-r_{32}\right) \\
\arccos \left(r_{33}\right) \\
\arctan 2\left(r_{13}, r_{23}\right)
\end{array}\right]
$$

which is used for computing projected areas and the angle of attack of the fluid. Note, that arccos of \pm 1 is not defined. The arccos function returns the angle between 0 and $\pi$ radians. The singularities are identified with Eq. (21) as $r_{33}=-1 \rightarrow \pi$ radians and $r_{33}=1 \rightarrow 0$ radians.

A common problem in the integration of rotational motions described by quaternions is the inherent quaternion drift. The quaternion is defined as a unit quaternion and its length has to remain equal to one. However, during integration the quaternions will drift away from this unit length. Therefore, it is necessary to re-normalize the quaternions at an appropriate time.

$$
q_{\text {new }}=\frac{q}{|q|},|q|=\sqrt{q_{0}^{2}+q_{1}^{2}+q_{2}^{2}+q_{3}^{2}}
$$

During the particle simulation the normalization was performed after each completed time step.

Finally, solving the equation for the particle angular velocity, Eq. (6) and Eq. (17) may be rewritten as

$$
\dot{\vec{\omega}}_{p}=\mathrm{I}_{p}^{-1}\left(\vec{T}-\vec{\omega}_{p} \times\left(\mathrm{I}_{p} \vec{\omega}_{p}\right)\right)
$$

Introducing a reference moment of inertia, the moments of inertia may be non-dimensionalized

$$
I_{\mathrm{ref}, p}=\rho_{p} d_{p}^{5}, \quad I_{p}^{\prime}=\frac{I_{p}}{I_{\mathrm{ref}, p}}
$$

which equates for spheres and cylinders to

$$
\begin{aligned}
I_{p, \text { sphere }}^{\prime} & =\frac{\pi}{60} \\
I_{p, x, \mathrm{cyl}}^{\prime} & =\frac{\pi}{32} c, \quad I_{p, y, z, \mathrm{cyl}}^{\prime}=\frac{1}{48} c\left(\frac{3}{4}+c^{2}\right)
\end{aligned}
$$

the dimensionless angular acceleration yields

$$
\begin{aligned}
& \dot{\vec{\omega}}_{p}^{\prime}=\left(\mathrm{I}_{p}^{\prime}\right)^{-1}\left(\vec{T}^{\prime}-\vec{\omega}_{p}^{\prime} \times\left(\mathrm{I}_{p}^{\prime} \vec{\omega}_{p}^{\prime}\right)\right) \\
& \vec{\omega}_{p}^{\prime}=\vec{\omega}_{p} \frac{L_{\mathrm{ref}}}{\dot{x}_{\mathrm{ref}}}
\end{aligned}
$$

$\dot{x}_{\text {ref }}$ equals the magnitude of the free stream velocity and $L_{\mathrm{ref}}$ is the characteristic length of the airfoil, e.g. the chord length. These are equations for the translational and angular acceleration and the quaternion. It represents a system of 10 differential equations to be solved instead of 3 differential equations to be solved for the basic Lagrangian trajectory simulation. The equations are integrated in time numerically with an embedded fourth-order Runge-Kutta method.

\section{Forces and Torques}

The sum of all forces in Eq. (3) acting on the particle may consist of the aerodynamic lift and drag and forces resulting from the density difference between particle and fluid (buoyancy and gravity),

$$
\sum \vec{F}=\vec{F}_{\text {Drag }}+\vec{F}_{\text {Lift }}+\vec{F}_{\text {Buoyancy }}+\vec{F}_{\text {Gravity }}+\cdots .
$$

All other forces are neglected. Introducing the relative velocity between particle and fluid $\overrightarrow{\dot{x}}_{\text {rel }}=\overrightarrow{\dot{x}}_{p}-\overrightarrow{\dot{x}}_{f}$ and using a drag law, where the drag varies with the relative velocity squared, Eq. (3) may be written

$$
\begin{aligned}
& \overrightarrow{\ddot{x}}_{p}=\left(-C_{D}+C_{L} R\left(q_{\perp}\right)\right) \frac{A_{p, S} \rho_{f}}{2 m_{p}}\left|\overrightarrow{\dot{x}}_{\mathrm{rel}}\right| \overrightarrow{\dot{x}}_{\mathrm{rel}}-\frac{\rho_{f}}{\rho_{p}} \vec{g}+\vec{g}, \\
& q_{\perp}:=\left(\frac{\pi}{2}, \vec{a}_{\mathrm{rot}}\right) \rightarrow R\left(q_{\perp}\right), \\
& \vec{a}_{\mathrm{rot}}:= \begin{cases}\text { if } \frac{\overrightarrow{\vec{x}}_{\mathrm{rel}} \cdot \vec{z}}{\left|\overrightarrow{\vec{x}_{\mathrm{rel}}} \cdot \vec{z}\right|}=1 & \text { then }[0,-1,0]^{T} \\
\text { if } \frac{\vec{x}_{\mathrm{rel}} \cdot \vec{z}}{\left|\vec{x}_{\mathrm{rel}} \cdot \vec{z}\right|}=-1 & \text { then }[0,1,0]^{T}, \\
& \text { else } \frac{\vec{x}_{\mathrm{re}} \times \vec{z}}{\left|\vec{x}_{\mathrm{rel}} \times \vec{z}\right|}\end{cases}
\end{aligned}
$$

where $A_{p, S}, \rho_{p}, m_{p}$ and $C_{D}$ are the cross section of the equivalent sphere, the particle density, the mass and the drag coefficient of the particle, respectively. $\rho_{f}$ is the density of the fluid. $\vec{g}$ is the gravity vector. The drag force 
is negative parallel and the lift force acts perpendicular to the relative velocity vector. The orientation of the lift force can be established by applying a Householder transformation, arbitrary axis is chosen to be the body fixed $\vec{z}:=[0,0,1]^{T}$ axis, and conversion into a quaternion to evaluate the rotation matrix $R\left(q_{\perp}\right)$.

By rearranging the equation above, Eq. 34 may be expressed as

$$
\overrightarrow{\ddot{x}}_{p}=\left(-C_{D}+C_{L} R\left(q_{\perp}\right)\right) \frac{\operatorname{Re}_{p}}{24} \frac{18}{d_{p}^{2}} \frac{\mu_{f}}{\rho_{p}} \overrightarrow{\dot{x}}_{\mathrm{rel}}+\frac{\rho_{p}-\rho_{f}}{\rho_{p}} \vec{g} .
$$

with $d_{p}$, the particle diameter, and $\mathrm{Re}_{p}$, the particle Reynolds number, defined as

$$
\operatorname{Re}_{p}=\frac{\left|\overrightarrow{\dot{x}}_{\mathrm{rel}}\right| d_{p} \rho_{f}}{\mu_{f}}
$$

If Eq. 35 is written in dimensionless form (dashed quantities are dimensionless),

$$
\overrightarrow{\ddot{x}}_{p}^{\prime}=\left(-C_{D}+C_{L} R\left(q_{\perp}\right)\right) \frac{\operatorname{Re}_{p}}{24} \frac{1}{K} \overrightarrow{\dot{x}}_{\mathrm{rel}}^{\prime}+\operatorname{Fr}^{2} \frac{\rho_{p}-\rho_{f}}{\rho_{p}} \frac{\vec{g}}{|\vec{g}|}
$$

then two similarity parameters of the particle-fluid interaction become apparent, the so-called inertia parameter $K$,

$$
K=\frac{d_{p}^{2}}{18} \frac{\rho_{p}}{\mu_{f}} \frac{\dot{x}_{\mathrm{ref}}}{L_{\mathrm{ref}}}
$$

and the Froude number, $\mathrm{Fr}=\dot{x}_{\text {ref }} / \sqrt{|\vec{g}| L_{\text {ref }}}$.

The inertia parameter $K$ is the ratio between a particle relaxation time and a characteristic time of the fluid flow. The Froude number Fr is the ratio between gravitational forces and fluid forces.

Hölzer and Sommerfeld [15] introduced a drag correlation for the complete Reynolds number region:

$$
\begin{aligned}
C_{D} & :=\frac{8}{\operatorname{Re}_{p}} \frac{1}{\sqrt{\Phi_{\|}}}+\frac{16}{\operatorname{Re}_{p}} \frac{1}{\sqrt{\Phi}}+\frac{3}{\sqrt{\operatorname{Re}}} \frac{1}{\Phi^{\frac{3}{4}}}+ \\
& +0.42 \cdot 10^{0.4(-\log (\Phi))^{0.2}} \frac{1}{\Phi_{\perp}}
\end{aligned}
$$

which depends on the shape, the orientation and the particle Reynolds number. The sphericity $(\Phi)$ represents the ratio between the surface area of the volume equivalent sphere and that of the considered particle. The crosswise sphericity $\left(\Phi_{\perp}\right)$ is the ratio between the crosssectional area of the volume equivalent sphere and the projected cross-sectional area of the considered particle. The lengthwise sphericity $\left(\Phi_{\|}\right)$is the ratio between the cross-sectional area of the volume equivalent sphere and the difference between half the surface area and the mean projected longitudinal cross-sectional area of the considered particle.

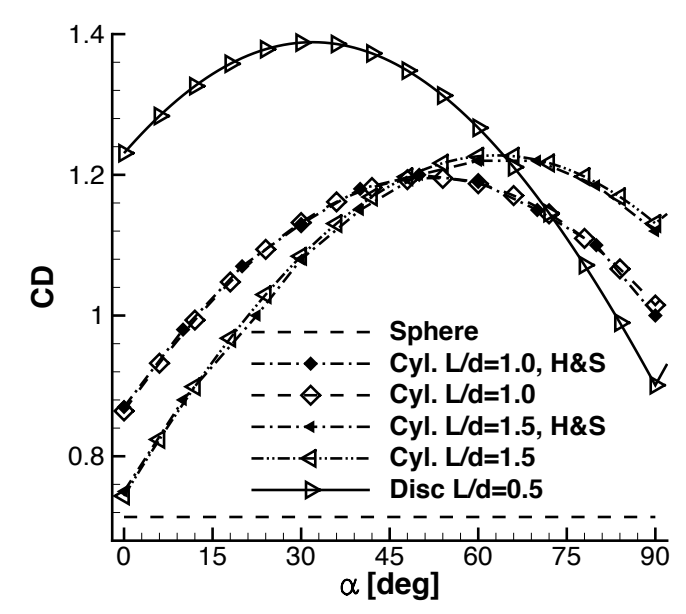

Figure 2: Comparison of the values for drag coefficient extracted from Hölzer and Sommerfeld [19] for cylindrical particles at $\operatorname{Re}_{p}=240$ with the implementation in TAU. In addition, the drag coefficient for a disc with an aspect ratio of 0.5 is presented.

The drag coefficient for cylindrical particles obtained from Hölzer and Sommerfeld is presented in Figure 2 in comparison with the implementation of Eq. 39 in TAU for a particle Reynolds number of 240 for a quarter rotation of the particle. Figure 2 includes the drag coefficient for a disc with an aspect ratio of 0.5 . A noticeable effect is the higher drag coefficient for the disc at 90 degrees (flat in flow direction) and the lower coefficient for the cylindrical particle at 0 degree (cross section in flow direction). The diameter was kept constant which then scales the length or thickness of the particle with the aspect ratio.

To characterize non-spherical objects, Wadell [20] introduced the sphericity $\Phi$. It is the ratio of a volumetric equivalent surface of a sphere and the actual surface of a particle. The sphericity is defined as

$$
\begin{gathered}
\Phi=\frac{A_{0, \text { Sphere }}}{A_{0}}:=\frac{d_{v}^{2}}{d_{A_{0}}^{2}}, \quad d_{A_{0}}=\sqrt{\frac{1}{\pi} A_{0}}, \\
\Phi_{\text {cyl }}=\frac{2\left(\frac{3}{2} c\right)^{2 / 3}}{1+2 c}, \quad c=\frac{L}{d_{p}}, \quad d_{v}:=\left(\frac{6}{\pi} V_{p}\right)^{1 / 3}
\end{gathered}
$$

$\Phi$ will be in the range $\Phi \leq 1$, with $\Phi \equiv 1$ for a sphere and $d_{v}$ is the equivalent diameter for a sphere of the same volume. Obviously, the sphericity does not account for orientation of the particle which is evident and was noted already by Wadell.

Similar to the sphericity, two additional sphericities may be defined, the crosswise and lengthwise sphericity, re- 
spectively,

$$
\begin{aligned}
\Phi_{\perp} & :=\frac{A_{\perp, \text { Sphere }}}{A_{\perp}}:=\frac{d_{v}^{2}}{d_{A_{\perp}}^{2}}, d_{A_{\perp}}:=\sqrt{\frac{4}{\pi} A_{\perp}} \\
\Phi_{\|} & :=\frac{A_{\|, \text {Sphere }}}{\frac{1}{2} A_{0}-A_{\|}}:=\frac{d_{v}^{2}}{2 d_{A_{0}}^{2}-d_{A_{\|}}^{2}}, \quad d_{A_{\|}}:=\sqrt{\frac{4}{\pi} A_{\|}}
\end{aligned}
$$

In general the crosswise sphericity is much easier to evaluate then the lengthwise sphericity. Replacing the lengthwise with the crosswise sphericity in the general correlation formula Eq. (39) will result in a small relative deviation compared to the general formula as mentioned by Hölzer and Sommerfeld [15].

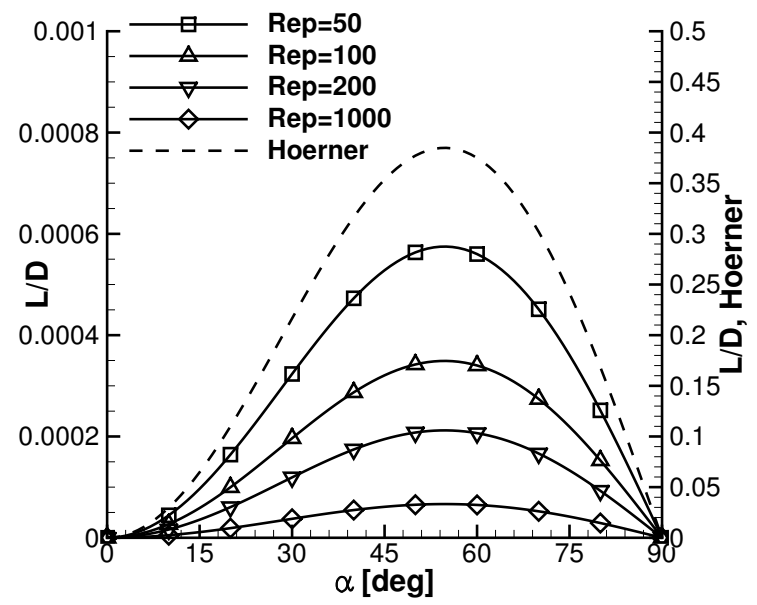

Figure 3: Lift over drag coefficient ratio from Mandø and Rosendahl [10] and Hörner [21] for a quarter rotation of the particle.

Lift forces may be differentiated into aerodynamic lift, arising from circulation generated in the fluid flow around the particles shape, as well as lift due to velocity gradients in the flow and lift due to particle rotation. The latter two are usually called Saffmann and Magnus lift-force, respectively. In this paper, aerodynamic lift is considered only. Aerodynamic lift may be modelled based on the cross-flow principle of Hörner [21] which relates the aerodynamic drag coefficient with the lift coefficient by the incidence angle

$$
\frac{C_{L}}{C_{D}}=\sin ^{2} \alpha \cos \alpha, \quad 0 \leq \operatorname{Re}_{p} \leq 10^{3}
$$

Mandø and Rosendahl [10] modified the ratio between lift and drag coefficient to also depend on the particle Reynolds number

$$
\frac{C_{L}}{C_{D}}=\frac{\sin ^{2} \alpha \cos \alpha}{0.65+40 \operatorname{Re}_{p}^{0.72}} .
$$

Figure 3 compares both approaches. Obviously, Mandø and Rosendahl's relationship is about three orders of magnitude smaller than Hörners approach. Due to that discrepancy, Eq. (44) is implemented.

The sum of all torques in Eq. 6),

$$
\sum \vec{T}=\vec{T}_{c . p .}+\vec{T}_{R}+\ldots
$$

are torques around the center of pressure as aerodynamic forces appear and the particles resistance torque against rotation. All other torques are neglected. Aerodynamic forces act around the center of pressure (c.p.) at the length $x_{c . p \text {. }}$ from the center of gravity. The resulting torque is

$$
\vec{T}_{c . p .}=\vec{x}_{c . p .} \times\left(R_{g a} \vec{F}_{D}+R_{g a} \vec{F}_{L}\right)
$$

with the transformation matrix $R_{g a}$ from the aerodynamic into the geodesic frame. As the particle is not discretized itself, an adequate description of $x_{c . p .}$. see Figure 1 , is extracted from the literature. Rosendahl [8] describes $x_{c . p .}$. as a function of the incidence angle $\alpha$ and the aspect ratio of axes $c=L / d$

$$
\frac{x_{c . p .}}{L}=\frac{1}{2}(1-\exp (1-c))\left(1-\sin ^{3} \alpha\right)
$$

and his co-worker Yin [22] proposed a similar relationship

$$
\frac{x_{c . p .}}{L}=0.25\left(1-\left|\cos ^{3} \alpha\right|\right)
$$

Both distributions are displayed in Figure 4 for a quarter rotation, indicating the orientation of the particle.

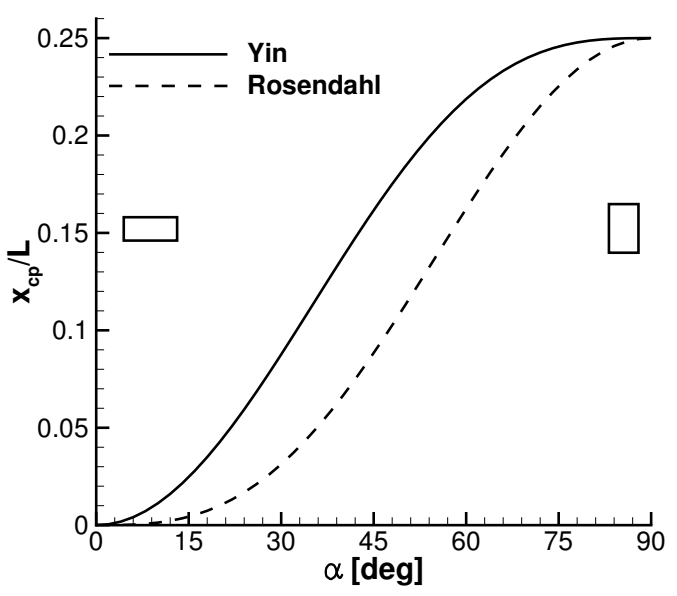

Figure 4: $x_{c . p .} / L$ location during rotation with indication of the body's orientation.

Throughout the simulations Yin's relationship of $x_{c . p .} / L$ was preferred. 


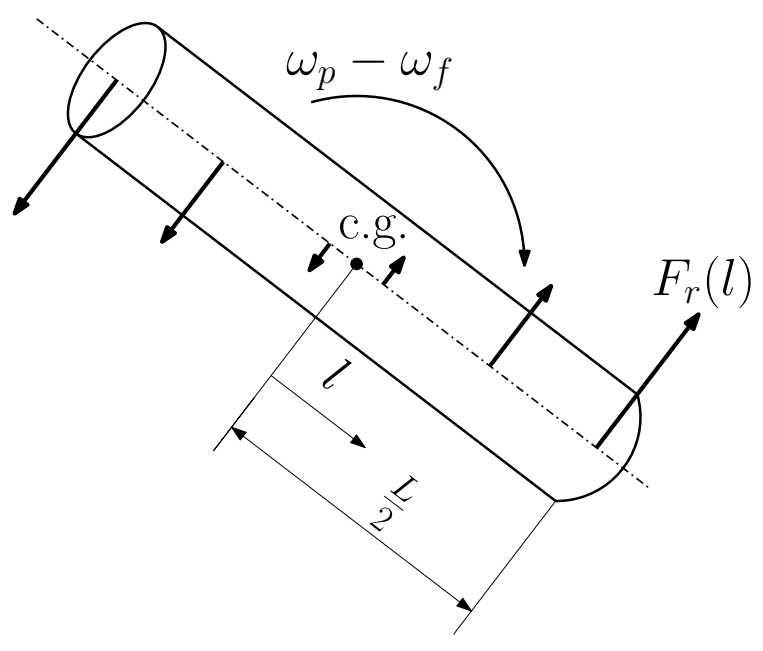

Figure 5: Resistance towards rotation [10].

The torque due to resistance can be directly derived by integration of the friction, caused by rotation, over the length of the particle, see Figure 5. First the torque will be derived with the simplification that the rotation takes place around the $y$-axis only, a two-dimensional simplification. The rotational velocity, the Reynolds particle number and the projected area are defined by

$$
\begin{aligned}
\vec{u}_{R} & =\vec{\omega}_{\text {rel }} \times \vec{l}, \quad \vec{\omega}_{\text {rel }}=\frac{1}{2} \vec{\omega}_{f}-\vec{\omega}_{p} \\
\operatorname{Re}_{R}(l) & =\frac{\rho_{f}\left|\vec{\omega}_{\text {rel }}\right| d_{p} l}{\mu}, \\
A_{r}(l) & =d l,
\end{aligned}
$$

where $A_{r}(l)$ denotes the projected area towards the particle rotation. White [23] found a drag correlation for cylinders in a uniform flow

$$
C_{D}(l) \approx 1+\frac{10}{\operatorname{Re}_{R}^{2 / 3}(l)}, 10^{-4}<\operatorname{Re}_{R}<2 \cdot 10^{5}
$$

which can be integrated analytically. Introducing that equation for the resistance torque, we obtain for a twodimensional rotation around the $y$-axis and introducing the aspect ratio $c=L / d_{p}$ in dimensionless form:

$$
\begin{aligned}
T_{r, y}^{\prime} & =\frac{T_{r, y}}{I_{\mathrm{ref}, p}}\left(\frac{L_{\mathrm{ref}}}{\dot{x}_{\mathrm{ref}}}\right)^{2}, \\
T_{r, y}^{\prime} & =\frac{\rho_{f}}{\rho_{p}} \frac{\left(\omega_{p, y}-\omega_{f, y}\right)^{2} c^{4} L_{\mathrm{ref}}^{2}}{\dot{x}_{\mathrm{ref}}^{2}}\left(\frac{1}{64}+\frac{1}{3.36 \mathrm{Re}_{R}^{2 / 3}}\right)
\end{aligned}
$$

Figure 6 points out the rotational torque coefficients for different cylindrical shapes using Eq. (54). An analytical expression derived for the rotational torque by Dennis [24], $T_{r}^{\prime}=6.84 / R e_{R}^{1 / 2}+31 / R e_{R}$ is depicted as well. Note that Eq. (54) is non-dimensionalized as proposed by Dennis to display them accordingly.

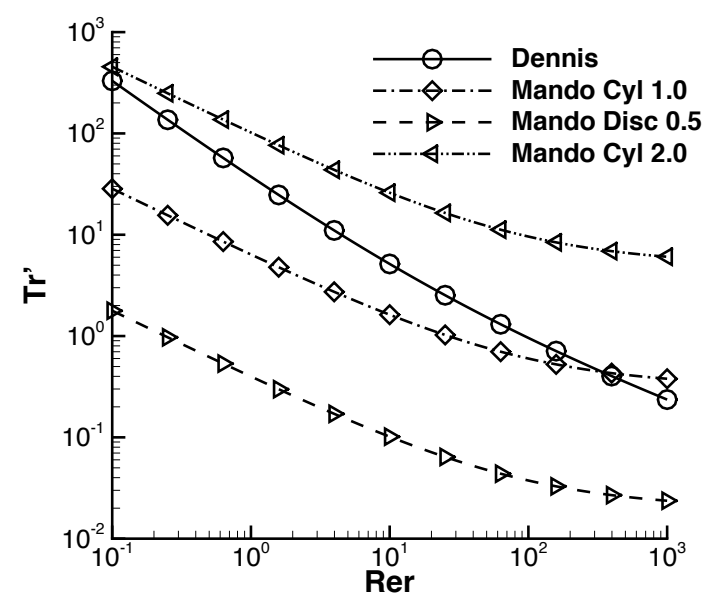

Figure 6: Rotational torque coefficient $T_{r}^{\prime}$ as a function of the rotational particle Reynolds number for the different approaches, Dennis [24] correlation for a sphere is used as a reference.

A more recent approach for the rotational torque has been presented by Zastawny et. al. [25] based on Direct Numerical Simulations (DNS) for prolate/oblate spheroids, discs and fibers. The resulting formula is a curve-fit taking into account symmetric and anti-symmetric axis of the particles shape. However, the implementation follows Eq. (54).

\section{Initial conditions}

Initialization of the particle's initial location is provided through user input via a single start point, a line of start positions or a two-dimensional equidistant grid of start positions. After specifying the release location, the particle velocity is set to the interpolated fluid velocity

$$
\left.\overrightarrow{\dot{x}}_{p}\right|_{t=0}=\left.\overrightarrow{\dot{x}}_{f}\right|_{t=0} .
$$

Regarding rotation, the initial Euler angles $\vec{\varphi}_{p}$ are set to $[0, \pi / 4,0]^{T}$. Due to the fact that the center of pressure location equals zero at $\theta \equiv 0$, an initial value $\theta \neq 0$ has to be taken. Otherwise no rotation of the particle occurs.

Setting the initial particle angular velocity is very difficult, because there is barely any information or experimental data available regarding the angular velocity distribution at flight altitudes.

In opposition to the numerical modeling of the flow past an object a uniform flow is assumed at the far field. Moreover the rotation of the particle is driven with the torque caused by aerodynamic forces and the viscous resistance against rotation. However, as the equilibrium torque between aerodynamic and resistance torque in a uniform flow is not known a priori, the particle release location is 
well ahead of the obstacle to ensure an almost constant particle angular velocity.

Ice density of ice particles is usually assumed to be 914 $\mathrm{kg} / \mathrm{m}^{3}$, which corresponds to the ice density of glace ice.

\section{Local collection efficiency}

The local collection efficiency $\beta$, is the ratio of the area $A_{0}$ spanned by four particle release points in the release plane projected on to a plane perpendicular to the oncoming flow and the area $A_{m}$ spanned by the corresponding particle impingement points, see Figure 7, and defined by

$$
\beta=\frac{A_{0} \cos \alpha}{A_{m}}
$$

where $\alpha$ is the angle of attack of the flow. The calculated $\beta$ value is assigned to the centroids $(+)$ of the quadrilaterals.

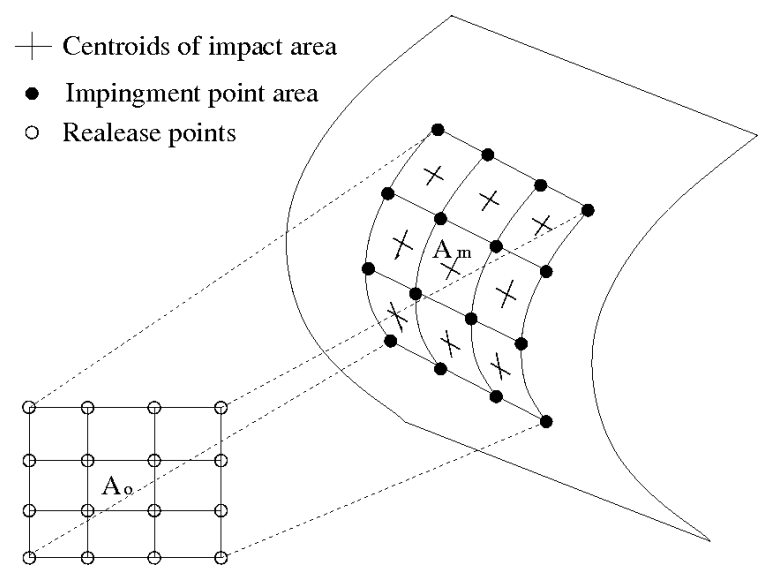

Figure 7: Determination of local catch efficiency $\beta$ for three-dimensional droplet impingement.

\section{Results}

It is difficult to validate the correct implementation of the afore-mentioned equations of motion and the physical correctness of the force and torque correlations: Experimental data are rare or do not fit to the flow conditions of interest and DNS simulations can not be performed because of computational cost. However, to gain a certain confidence in the implementation, Hölzer and Sommerfeld's drag correlation model has been compared to the original data set, see Figure 2, and the rotational torque of particles was compared against analytical derived formulas, Figure 6. Moreover, to avoid implementation errors, for computing the torque generated by aerodynamic forces a procedure is used as a template that has been already in use for many years in the TAU code for calculation of airfoil pitching moments.
Therefore, any test case presented in the following is not a validation of the implementation. The test cases only serve the purpose to show the appropriateness of a nonrotating particle initial condition and to illustrate the importance of taking into account the rotational motion of particles in certain flow situations. The following test cases have been considered: The first test case is a potential flow past a cylinder to investigate the development of particle rotation for particles released far upstream of the cylinder with a zero initial particle angular velocity. The second case is a subsonic laminar flow around a NACA0012 airfoil and the third case is a turbulent flow around a high-lift device. Both test cases are selected to determine collection efficiencies on the airfoils comparing rotating cylindrical particles and non-rotating spheres.

As it is common to describe the rotation behavior with similarity parameters, the particle spin number is introduced as follows

$$
\mathrm{Sp}=\frac{\omega_{p} d_{p}}{U_{f, \infty}}
$$

which is in an aerodynamic context the reduced frequency. Eq. (57) can be remodeled with the assumption

$$
\begin{aligned}
& \omega_{p}:=\omega_{p}^{\prime} \frac{\dot{x}_{\mathrm{ref}}}{L_{\mathrm{ref}}}, \omega_{p}^{\prime}=U_{f, \infty}^{\prime} \equiv \mathrm{Ma}_{\infty} \\
& \mathrm{Sp}=\frac{U_{f, \infty}^{\prime} \dot{x}_{\mathrm{ref}} d_{p}}{U_{f, \infty}^{\prime} \dot{x}_{\mathrm{ref}} L_{\mathrm{ref}}}=\frac{d_{p}}{L_{\mathrm{ref}}}
\end{aligned}
$$

where $\mathrm{Ma}_{\infty}$ denotes the freestream Mach number. In particle related literature, a reference particle diameter is often defined as $L_{\text {ref }} \equiv d_{p \text {,ref, mainly for use in non- }}$ dimensionalization. Thus, the spin number becomes unity.

\section{Flow past a cylinder}

The first test case, a potential flow past a cylinder, has been selected to demonstrate the rotation behavior of particles. The cylindrical body considered has a diameter of $0.5 \mathrm{~m}$ and a circular far field encompasses the cylinder at a radius of $4.5 \mathrm{~m}$. The flow conditions are summarized in Table 1.

Table 1: Numerical Simulation parameter for the flow past a Cylinder

\begin{tabular}{ccccc}
\hline $\mathrm{Ma}_{\infty}$ & $\alpha$ [deg.] & $\dot{x}_{\text {ref }}[\mathrm{m} / \mathrm{s}]$ & $d_{\text {cyl }}[\mathrm{m}]$ & $d_{p}[\mu \mathrm{m}]$ \\
\hline 0.3 & 0.0 & 277.4 & 0.5 & 100 \\
\hline
\end{tabular}

Cylindrical particles are released very close to the upstream far field boundary with an initial particle angular velocity of zero without considering gravity forces. Two trajectories are considered with different particle aspect 
ratios, passing by very close to the top and bottom of the cylinder. The trajectories of the particle with aspect ratio of $L / d=10$ can be seen in Figure 8 and for an aspect ratio of $L / d=0.1$ in Figure 9 as dashed lines. The trajectories marked by solid lines, in both figures, are the same particles, but without considering rotation, serving as a reference. The flow field in both figures is contoured with the static pressure.

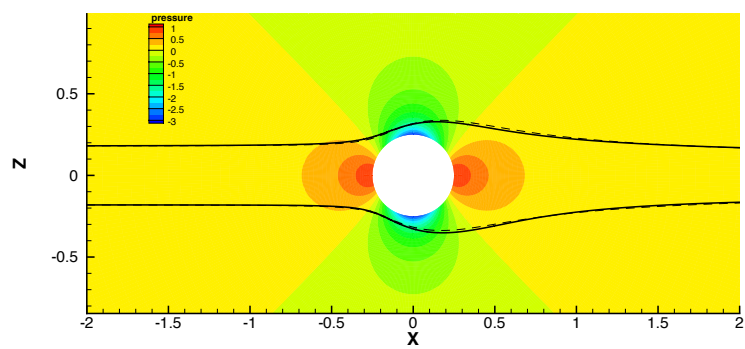

Figure 8: Flow past a cylinder at $\mathrm{Ma}_{\infty}=0.3$ showing the trajectory of a cylindrical particle with aspect ratio $L / d_{p}=$ $10\left(d_{p}=100 \mu \mathrm{m}\right)$ without rotation (solid) and with selfinduced rotation (dashed).

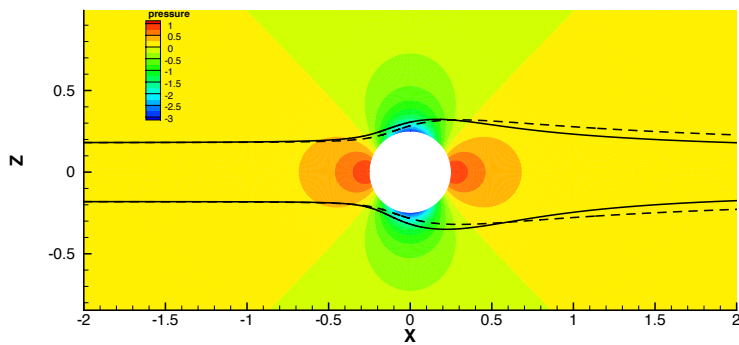

Figure 9: Flow past a cylinder at $\mathrm{Ma}_{\infty}=0.3$ showing the trajectory of a cylindrical particle with aspect ratio $L / d_{p}=$ $0.1\left(d_{p}=100 \mu \mathrm{m}\right)$ without rotation (solid) and with selfinduced rotation (dashed).

The upper and lower trajectory between rotating and nonrotating particles with an aspect ratio $L / d_{p}=10$ do not differ much as seen in Figure 8. That is related to the overall low spin of the particles for $L / d_{p}>1$ seen in Figure 10 and Figure 11. The lifting force and torque will not have a great impact, except for the drag which remains nearly the same as for the non-rotating particle. Figure 9 displays the upper and lower trajectory for the rotating particle with $L / d_{p}=0.1$. It is much more widespread to its non-rotating counterpart and they are not symmetric to the $z \equiv 0$ line additionally. First, the deviation to rotating particles with $L / d_{p}>1$ is caused by its higher spinning rate while passing the obstacle in comparison to particles with $L / d_{p}<1$, resulting in a higher influence of the aerodynamic force.
Second, the non-symmetric behavior between both rotating particle trajectories is related to a negative angle of attack at the upper trajectory and a positive on the lower, respectively, but the rotation is unaffected by the angle of attack, see both Figure 10 and Figure 11 for corresponding aspect ratios.

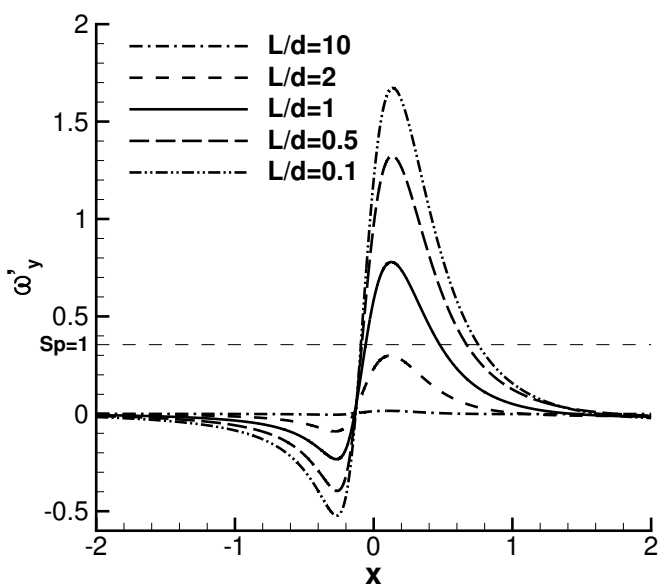

Figure 10: Dimensionless particle angular velocity monitored over covered distance for the trajectories passing the object above for various aspect ratios $L / d_{p}, d_{p}$ is 100 $\mu \mathrm{m}$.

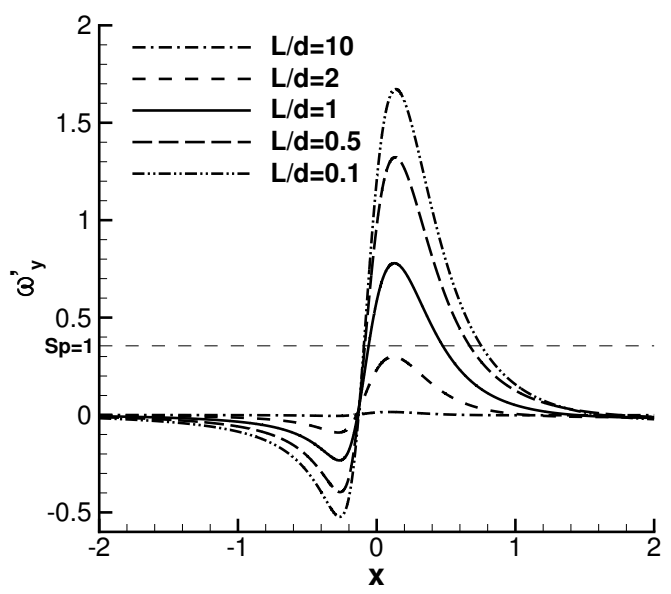

Figure 11: Dimensionless particle angular velocity monitored over covered distance for the trajectories passing the object below for various aspect ratios $L / d_{p}, d_{p}$ is 100 $\mu \mathrm{m}$.

The dimensionless angular velocity of the considered particles are plotted in Figure 10, related to the trajectories passing the obstacle above, and Figure 11 is related to 
the trajectories passing the obstacle below. Both figures seem to be the same. The difference in the angular velocity for the rotating particles is marginal between the trajectories passing above or below the obstacle. The spin of the particle increases naturally when approaching towards the obstacle. All particles experience a raise in spin while passing the obstacle and the rotation direction is reverted. The spin number of 1 is reached at $\omega_{p, y}^{\prime} \equiv \mathrm{Ma}_{\infty}=0.3$. The particles with higher aspect ratio are nearly unaffected because the moment of inertia is increasing quadratically with the particle dimension. A cylindrical particle with aspect ratio 10 has an equivalent diameter of $380 \mu \mathrm{m}$ while an aspect ratio of 0.1 results in an equivalent diameter of $66 \mu \mathrm{m}$. After about three times the obstacles diameter, the particle angular velocity has decayed almost to zero.

\section{NACA0012 subsonic laminar case}

The next test case was defined for the High Altitude Ice Crystal (HAIC) project [26] as the TRL4-2 benchmark case to investigate the dependence of the collection efficiencies $\beta$ on drag correlations, phase change and other relevant parameters for ice crystals. The flow conditions for the NACA0012 airfoil are summarized in Table 2.

Table 2: Numerical simulation parameters for the laminar flow around the NACA0012 airfoil.

\begin{tabular}{ccccc}
\hline $\mathrm{Ma}_{\infty}$ & $\operatorname{Re}_{\infty}\left[10^{6}\right]$ & $\alpha$ [deg.] & $\dot{x}_{\text {ref }}[\mathrm{m} / \mathrm{s}]$ & $L_{\text {ref }}[\mathrm{m}]$ \\
\hline 0.3 & 3.8 & 2.0 & 277.4 & 0.5 \\
\hline
\end{tabular}

Table 3: Particle simulation parameters for rotating cylinders and discs. The sphere is non-rotating. Notations have the following meaning: equivalent sphere diameter $\equiv d_{v}$, sphericity $\equiv \Phi$ and the crosswise sphericity $\equiv \Phi_{\perp}$.

\begin{tabular}{cccccc}
\hline Case & $d_{p}(\mu \mathrm{m})$ & $L / d_{p}$ & $d_{v}(\mu \mathrm{m})$ & $\Phi$ & $\Phi_{\perp}$ \\
\hline Sphere & 20 & 1 & 20 & 1 & 1 \\
Cyl. & 20 & 10 & 49.3 & 0.58 & 0.48 \\
Cyl. & 20 & 2 & 28.8 & 0.83 & 0.82 \\
Disc & 20 & 0.5 & 18.2 & 0.83 & 0.83 \\
Disc & 20 & 0.1 & 10.6 & 0.47 & 0.28 \\
\hline Sphere & 100 & 1 & 100 & 1 & 1 \\
Cyl. & 100 & 10 & 246.6 & 0.58 & 0.48 \\
Cyl. & 100 & 2 & 144.2 & 0.83 & 0.82 \\
Disc & 100 & 0.5 & 53.1 & 0.83 & 0.83 \\
Disc & 100 & 0.1 & 90.9 & 0.47 & 0.28 \\
\hline
\end{tabular}

Table 3 presents the test matrix defined for the different shapes to be investigated. In addition, the equivalent spherical diameter, sphericity and cross-wise sphericity are included. The simulation of non-rotating spheres is shown as a reference.

All cylindrical shaped particles are released with the same spin number of 0.0004 to keep the unsteadiness comparable. It is calculated from the particle diameter of $d_{p}=20 \mu \mathrm{m}$ and divided by the reference length of $0.5 \mathrm{~m}$. 100 particles are released from equidistant start positions near the far field boundary of the computational domain to calculate the collection efficiency. The start position of the trajectories is chosen such that the airfoil is enclosed completely by trajectories. Thus, only a few trajectories will pass above and below the airfoil.

Figure 12 shows the computational grid and the flow field for the NACA0012 airfoil. An extensive refinement of the grid at the nose region allows a proper resolution of the collection efficiency. Most Lagrangian-type particle tracers suffer from a rough predicted collection efficiency, whenever the airfoils surface discretization becomes too coarse.
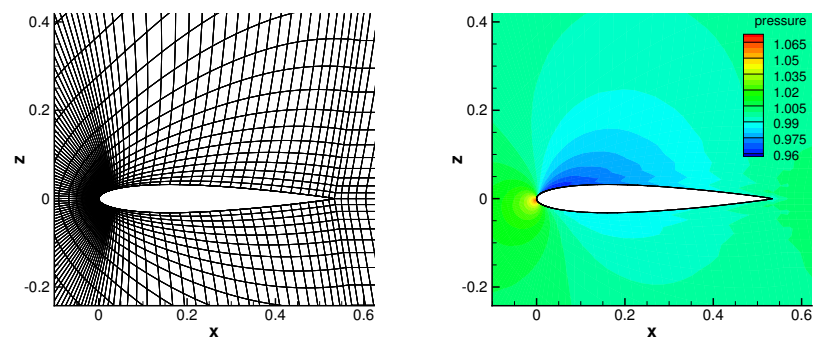

Figure 12: Computational grid of the NACA0012 airfoil and static pressure distribution of the flow simulation for $\mathrm{Ma}_{\infty}=0.3$ and angle of attack $\alpha=2$ deg. .

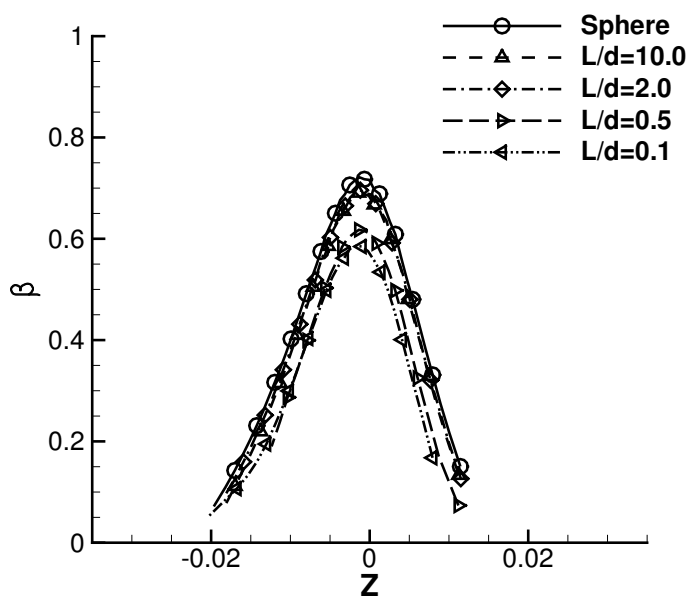

Figure 13: Collection efficiency $\beta$ on the NACA0012 airfoil for a particle diameter of $20 \mu \mathrm{m}$. 
The collection efficiency distribution over the Cartesian zcoordinate are displayed in Figure 13 and Figure 14 for the two particle diameters. In general, the distributions are almost indistinguishable from each other. An exception are the disc-shaped particles which have a collection efficiency clearly separated from the other particles. This separation reduces with increasing $d_{p}$. The same effect is also observed for spheres of the same equivalent diameter. A reason for the small deviation is the particles inertia response time. The relative velocity of the particle is low and therefore does not contribute much to the airfoils circulation, which is necessary to spin up or down the particle until it hits the surface of the airfoil.

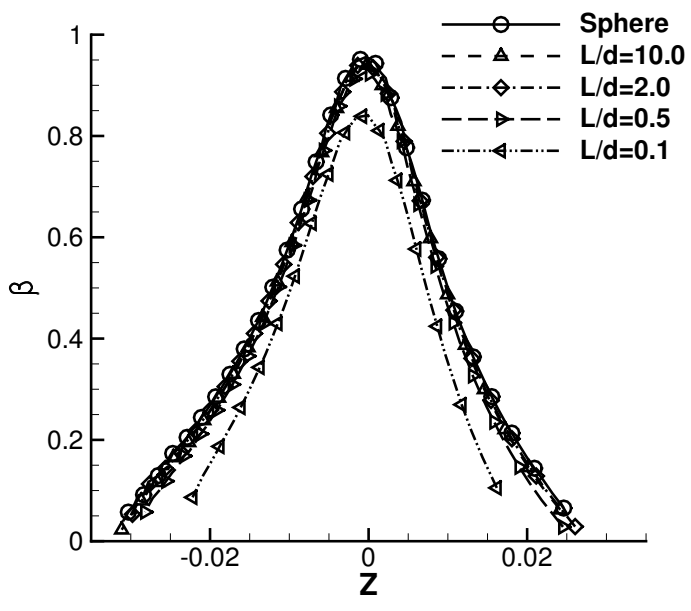

Figure 14: Collection efficiency $\beta$ on the NACA0012 airfoil for a particle diameter of $100 \mu \mathrm{m}$.

\section{High-lift three-element airfoil}

The third test case is the high-lift three-element airfoil NHLP L1/T2 [27]. Table 4 lists the subsonic turbulent flow simulation parameters. The high angle of attack and the design of the high-lift devices generate a strong circulation around the airfoil. Numerical simulations have been performed with the classic Spalart-Allmaras turbulence model [28]. For this test case, the interaction between the flow field and particles leads to strong excitation of the particle rotation since the flow vorticity (equivalent to the angular velocity of solid body rotation in the flow), $\omega_{f}$, is inherently linked to the particle angular velocity since it increases the relative angular velocity driving the rotation of the particle.
Table 4: Numerical simulation parameter for the NHLP L1/T2 high-lift three-element airfoil.

\begin{tabular}{ccccc}
\hline $\mathrm{Ma}_{\infty}$ & $\operatorname{Re}_{\infty}\left[10^{6}\right]$ & $\alpha$ [deg.] & $\dot{x}_{\text {ref }}[\mathrm{m} / \mathrm{s}]$ & $L_{\text {ref }}[\mathrm{m}]$ \\
\hline 0.197 & 3.52 & 4.0 & 279.99 & 1.0 \\
\hline
\end{tabular}

The computational grid, Figure 15, is entirely composed of quadrilaterals with a rectangular far field set 50 chord lengths away from the airfoil in both directions.

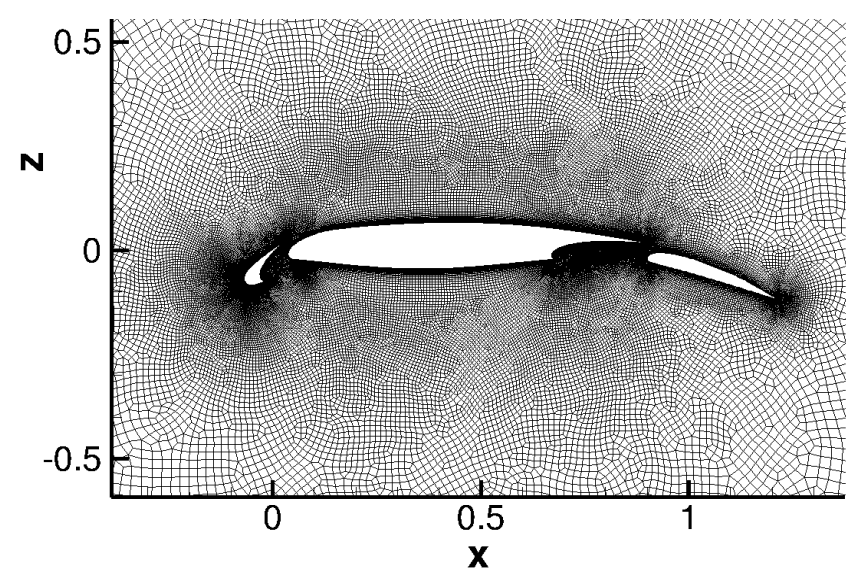

Figure 15: Computational grid for the NHLP L1/T2 high-lift three-element airfoil.

The close-up view of the slat-airfoil and airfoil-flap intersection can be seen in Figure 16. At both gaps the flow is accelerated, resulting in strong and varying flow velocity gradients.
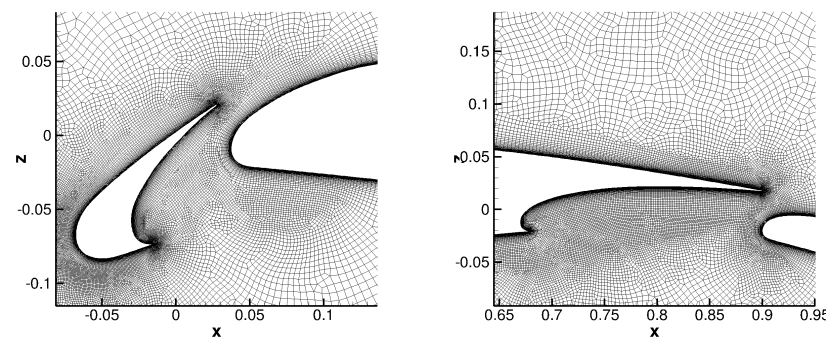

Figure 16: Detailed slat-airfoil and airfoil-flap region of the computational grid for the NHLP L1/T2 high-lift threeelement airfoil.

This effect is well seen in the contour plot of the Mach number in Figure 17. At the front gap, the flow velocity is approximately doubled from underneath the airfoil through the upper side. It is also obvious, that two recirculation regions appear at the back of the slat and main airfoil element with low flow velocities. 


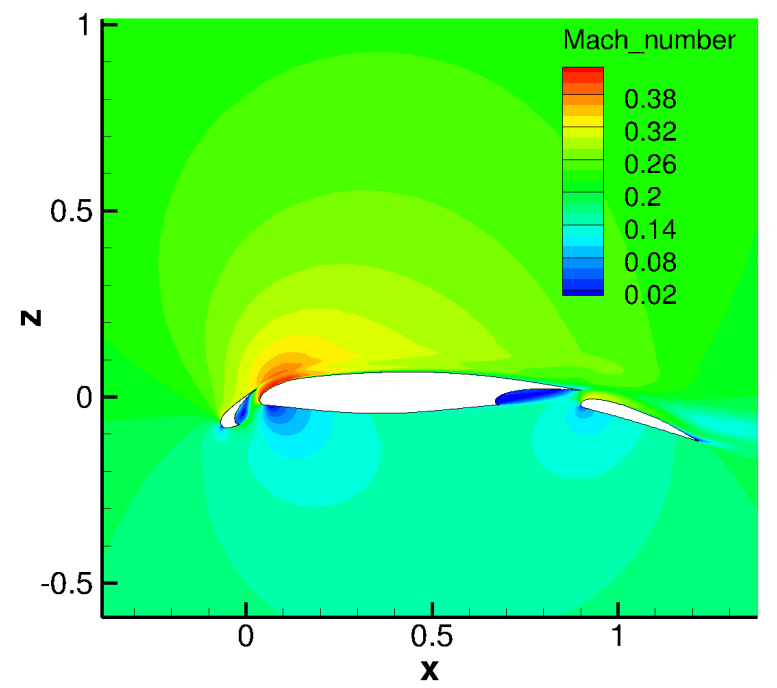

Figure 17: Flow field simulation of the NHLP L1/T2 highlift three-element airfoil at $\mathrm{Ma}_{\infty}=0.197, \mathrm{Re}_{\infty}=3.52$ million and $\alpha=4.0$ deg. .

Collection efficiencies have been obtained for the same particles as described in Table 3. $\beta$ distributions are presented over the Cartesian z-coordinate for the slat in Figure 18 and Figure 19, for the main airfoil element in Figure 20 and Figure 21 and for the flap in Figure 22 and Figure 23

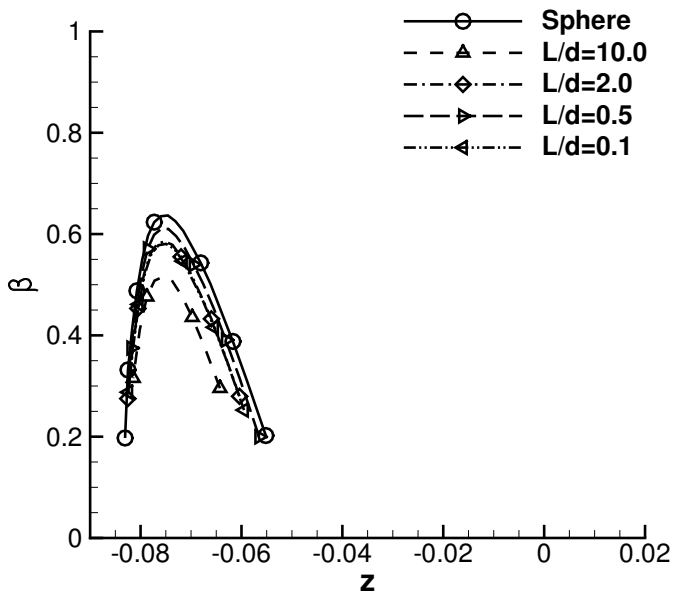

Figure 18: Collection efficiency distribution for a particle diameter of $20 \mu \mathrm{m}$ and various aspect ratios obtained for the slat.

For each element the collection efficiency for $20 \mu \mathrm{m}$ and $100 \mu \mathrm{m}$ particles are depicted in the left and right graph of these figures. The release location of all particles is near the far field boundary with 200 equidistant start points, flowing around the airfoil in a narrow band. The initial spin number is $\mathrm{Sp}=0.0002$ based on the particle diameter of $20 \mu \mathrm{m}$.

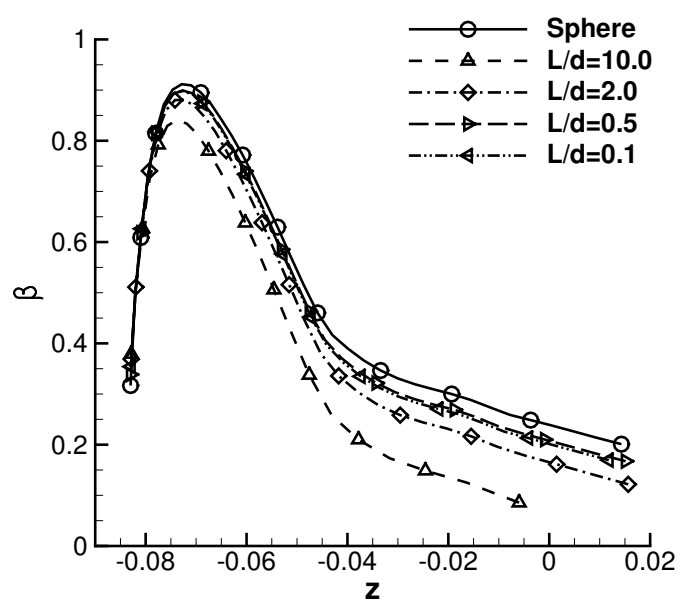

Figure 19: Collection efficiency distribution for a particle diameter of $100 \mu \mathrm{m}$ and various aspect ratios obtained for the slat.

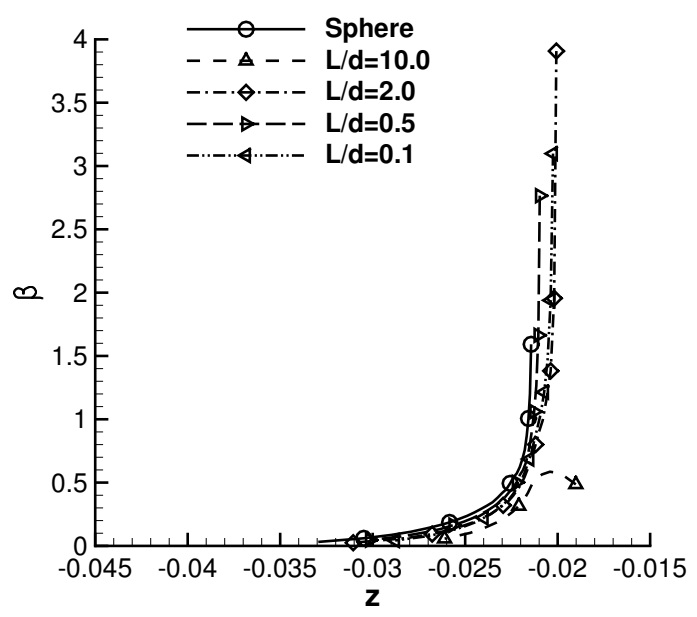

Figure 20: Collection efficiency distribution for a particle diameter of $20 \mu \mathrm{m}$ and various aspect ratios obtained for the airfoil main element.

The $\beta$ distribution for the slat in Figure 18 for a particle diameter of $20 \mu \mathrm{m}$ and in Figure 19 for $100 \mu \mathrm{m}$, respectively, shows a coincidence of graphs to the left of the airfoils stagnation point $(z \approx-0.075)$, which is equivalent to the pressure side of the slat, and a separation of graphs with a noticeable difference in $\beta$ to the right side (corresponding to the suction side). This behavior is caused by the blunt nose of the slat and the flow being accelerated 
on the suction side. Surprisingly, no particles reached the recirculation region.

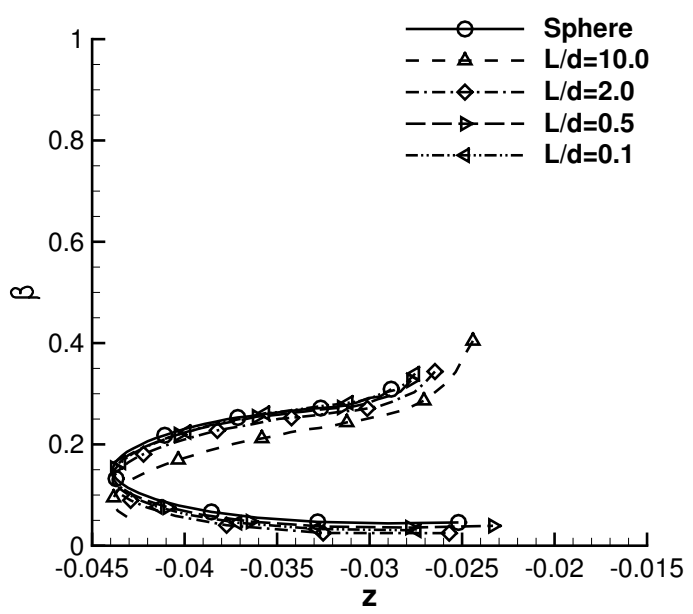

Figure 21: Collection efficiency distribution for a particle diameter of $100 \mu \mathrm{m}$ and various aspect ratios obtained for the airfoil main element.

The graph in Figure 20 for particle diameters of $20 \mu \mathrm{m}$ shows a $\beta$ exceeding a value of 1 for the sphere, a cylinder with $L / d_{p}=2$ and the disc with $L / d_{p}=0.5$. The reason for this behavior is the small gap between slat and main airfoil element. The stream tube formed by the particles initially released is compressed while sliding through the gap between slat and main airfoil element, resulting in a smaller impingement area. Otherwise, $\beta$ is different at the stagnation point of the main airfoil element only. A bit more challenging is the interpretation of the graph in Figure 21 for the particle diameter of $100 \mu \mathrm{m}$. The C-shaped distribution is caused by impingement on the whole bottom side of the main airfoil element. The upper part of the curve until the reversal point is from the stagnation point at the nose to the lowest $\mathrm{z}$-coordinate of the main airfoil element, which corresponds approximately to the mid-point of the airfoil on the lower side. The graph shows a narrow spread in the $\beta$ distribution, a reverted effect compared to the slat. Because of the angle of attack at the slat and main airfoil element, the exposed and shadow areas have reverted. The cylindrical particle with $L / d_{p}=10$ hits the bottom of the main airfoil element surface only until the minimum z-coordinate is reached.

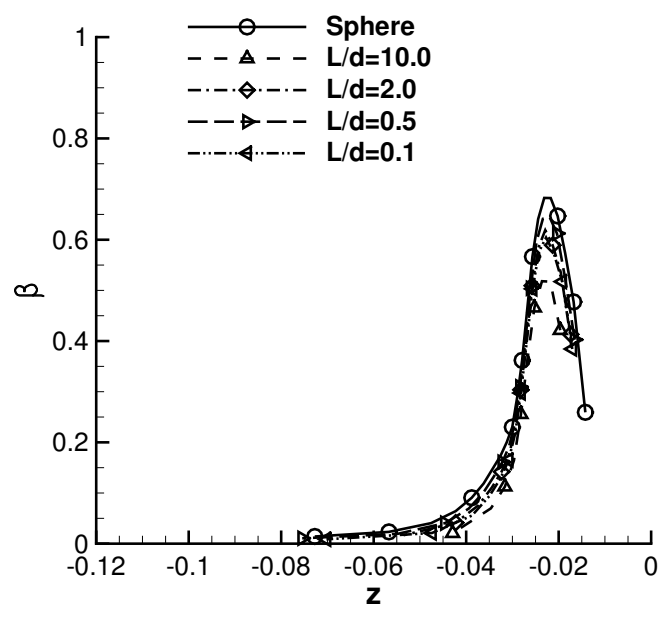

Figure 22: Collection efficiency distribution for a particle diameter of $20 \mu \mathrm{m}$ and various aspect ratios obtained for the flap.

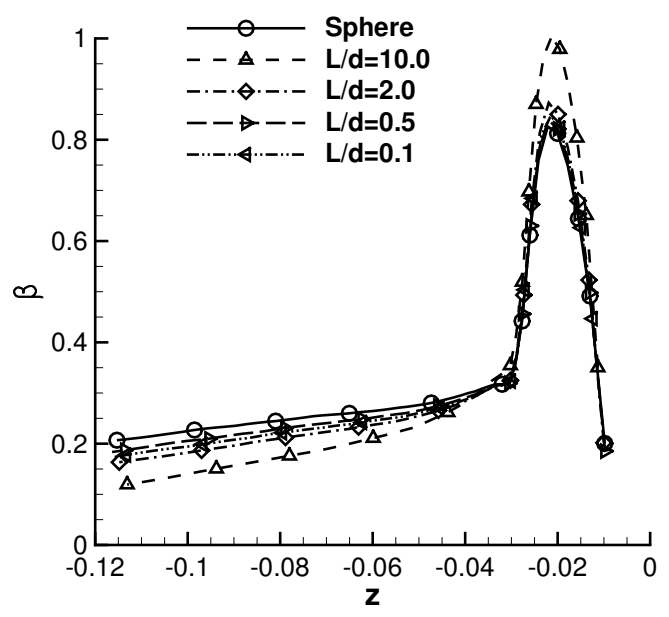

Figure 23: Collection efficiency distribution for a particle diameter of $100 \mu \mathrm{m}$ and various aspect ratios obtained for the flap.

The particle impingement on the flap, Figure 22 and Figure 23, is comparable to the main airfoil element since it is mainly impacted on the lower side. However, particles reach the upper side of the airfoil in a narrow band from the $\beta$-peak to the right end of the curves, which is visible in both figures. The maximum difference occurs in the region of the $\beta$ maximum and particularly the cylindrical particle with $L / d_{p}=10$ has a distinctly larger $\beta_{\max }$ compared to all other particle types.

Whenever it comes to industrial applications, the computational effort is often a measure for the applicability, results are given in Table 5. The simulation of spheres 
without rotation has been used as reference since it was the previous standard. The time ratio $\xi=t_{\text {rot }} / t_{\text {no-rot }}$ is the time for computing rotating particles divided by the time required for the non-rotating particles, each measured for 200 trajectories. The standard implementation has to solve six equations, three for the path and three for the velocity in a three-dimensional context. Now, for the rotation, seven additional equations need to be solved, four quaternion parameters and three for the particle angular velocity, which means that the number of equations to be solved is more than doubled.

Table 5: Computational effort for the trajectory simulation of the NHLP L1/T2 airfoil with the time factor $\xi=t_{\text {no-rot }} / t_{\text {rot }}$.

\begin{tabular}{lcccc}
\hline Particle-Shape & $L / d_{p}$ & $t_{\text {no-rot }}(\mathrm{s})$ & $t_{\text {rot }}(\mathrm{s})$ & $\xi$ \\
\hline $20 \mu \mathrm{m}$ & & & & \\
\hline Sphere & 1 & 10.54 & - & 1 \\
Cylinder & 10 & - & 45.05 & 4.3 \\
Cylinder & 2 & - & 45.53 & 4.3 \\
Cylinder & 0.5 & - & 47.39 & 4.5 \\
Cylinder & 0.1 & - & 50.21 & 4.8 \\
\hline $100 \mu \mathrm{m}$ & & & & \\
\hline Sphere & 1 & 5.69 & - & 1 \\
Cylinder & 10 & - & 18.35 & 3.2 \\
Cylinder & 2 & - & 17.62 & 3.1 \\
Cylinder & 0.5 & - & 19.49 & 3.4 \\
Cylinder & 0.1 & - & 20.87 & 3.7 \\
\hline
\end{tabular}

In summary, depending on the considered particle dimensions and flow conditions, an additional factor of three to four needs to be expected in terms of computing time if the influence of rotation plays an important role and needs to be considered.

\section{Conclusion}

A Lagrangian-type particle tracer based on the TAU Code has been presented, taking into account rotation of regular shaped particles. Validation of the implementation was based on a comparison of analytical solutions and literature based data and correlations for drag, forces and torques. However, no validation was possible using the standard way, e.g. by comparing trajectory paths or impingement distributions with experiments.

The influence of considering particle rotation on the collection efficiency is strongly linked to the application. It may be negligible for a transport aircraft cruising at high altitudes and transonic flow conditions with a moderately blunt nose. The situation changes significantly for airfoils or wings generating a high circulation as it is often required during take-off and landing at subsonic flow condi- tions. Particularly, in flow regions exposed to strong flow accelerations or decelerations as seen in the gaps between a slat and the main airfoil element and the main airfoil element and a flap or at the airfoil stagnation point.

\section{References}

[1] A. Haider, O. Levenspiel, Drag coefficient and terminal velocity of spherical and nonspherical particles, Powder Technology 58 (1) (1989) 63-70. doi : 10.1016/0032-5910(89)80008-7.

[2] G. H. Ganser, A rational approach to drag prediction of spherical and nonspherical particles, Powder Technology 77 (2) (1993) 143-152. doi:10.1016/ 0032-5910(93)80051-B

[3] S. Tran-Cong, M. Gay, E. E. Michaelides, Drag coefficients of irregularly shaped particles, Powder Technology 139 (2004) 21-32. doi:10.1016/j.powtec. 2003.10 .002

[4] G. B. Jeffery, The Motion of Ellipsoidal Particles Immersed in a Viscous Fluid, in: Proc. R. Soc. Lond. A, Vol. 102, 1922, pp. 161-179. doi:10.1098/rspa. 1922.0078

[5] R. G. Cox, The motion of long slender bodies in a viscous fluid: Part I General theory, J. Fluid Mech. 44 (4) (1970) 791-810. doi:10.1017/S002211207000215X

[6] D. Qi, Lattice-Boltzmann simualtions of particles in non-zero-Reynolds-number flows, J. Fluid Mech. 385 (1999) 41-62. doi : 10.1017/S0022112099004401.

[7] H. Huang, X. Yang, M. Krafczyk, X.-Y. Lu, Rotation of spheroidal particles in coutte flows, Journal of Fluid Mechanics 692 (2012) 369-394. doi:10.1017/jfm. 2011.519 .

[8] L. Rosendahl, Using a multi-parameter particle shape description to predict the motion of nonspherical particle shapes in swirling flow, Applied Mathematical Modelling 24 (1) (2000) 11-25. doi: 10.1016/S0307-904X (99)00023-2.

[9] E. Loth, Lift of a Solid Spherical Particle Subject to Vorticity and/or Spin, AIAA Journal 46 (2008) 801809. doi:10.2514/1.29159.

[10] M. Mandø, L. Rosendahl, On the motion of nonspherical particles at high Reynolds number, Powder Technology 202 (1-3) (2010) 1-13. doi:10.1016/j . powtec.2010.05.001.

[11] C. Kleinstreuer, Y. Feng, Computational Analysis of Non-Spherical Particle Transport and Deposition in 
Shear Flow With Application to Lung Aerosol Dynamics - A Review, Journal of Biomenchanic Engineering 135 (2013) 1-19. doi:10.1115/1.4023236.

[12] R. Zimmermann, Y. Gasteuil, M. Bourgoin, R. Volk, A. Pumir, J.-F. Pinton, Tracking the dynamics of translation and absolute orientation of a sphere in a turbulent flow, Review of Scientific Instruments 82 (033906) (2011) 1-9. doi:10.1063/1.3554304.

[13] M. Widhalm, A. Ronzheimer, J. Meyer, Lagrangian Particle Tracking on Large Unstructured ThreeDimensional Meshes, in: $46^{\text {th }}$ AIAA Aerospace Sciences Meeting and Exhibit, AIAA 2008-472, Reno, NV, 2008.

[14] I. Langmuir, K. B. Blodgett, U. S. A. A. Forces, A Mathematical Investigation of Water Droplet Trajectories, no. 5418 in Army Air Forces technical report, Army Air Forces Headquarters, Air Technical Service Command, 1946.

[15] A. Hölzer, M. Sommerfeld, New simple correlation formula for the drag coefficient of non-spherical particles, Powder Technology 184 (3) (2008) 361-365. doi: $10.1016 / j$. powtec . 2007.08 .021 .

[16] B. Kenwright, A beginners guide to dual-quaternion, in: V. Skala (Ed.), $20^{\text {th }}$ WSCG International Conference on Computer Graphics, Visualization and Computer Vision, Plzen, 2012.

[17] J. Diebel, Representing attitude: Euler angles, unit quaternions, and rotation vectors, University Lecture, Stanford University (2006).

[18] D. J. Evans, On the representation of orientation space, Mol. Phys. 34 (2) (1977) 317-325. doi: $10.1080 / 00268977700101751$.

[19] A. Hölzer, M. Sommerfeld, Lattice boltzmann simulations to determine drag, lift and torque acting on nonspherical particles, Computers \& Fluids 38 (2009) 572-589. doi:10.1016/j.compfluid.2008.06.001.

[20] H. Wadell, The Coefficient of Resistance as a Function of Reynolds Number for Solids of Various Shapes, Journal of the Franklin Institute 217 (4) (1934) 459-490. doi:10.1016/S0016-0032(34) 90508-1.

[21] S. F. Hörner, Fluid-Dynamic Drag, published by the author, 1965.

[22] C. Yin, L. Rosendahl, S. Knudsen, H. Sørensen, Modelling the motion of cylindrical particles in a nonuniform flow, Chemical Engineering Science 58 (15) (2003) 3489-3498. doi:10.1016/ S0009-2509(03) 00214-8
[23] F. M. White, Viscous fluid flow, 2nd Edition, McGrawHill, Inc., 1991.

[24] S. C. R. Dennis, D. B. Ingham, S. N. Singh, The steady flow of a viscous fluid due to a rotating sphere, Quarterly Journal of Mechanics and Applied Mathematics 34 (3) (1981) 361-373.

[25] M. Zastawny, G. Mallouppas, F. Zhoa, B. van Wachem, Derivation of drag and lift force and torque coefficients for non-spherical particles in flow, International Journal of Multiphase Flow 39 (2012) 227239. doi: j.ijmultiphaseflow.2011.09.004.

[26] F. D. A. Grandin, J.-L. Brenguier, F. Hervy, H. Schalger, P. Villedieu, G. Zalamansky, HAIC (High Altitude Ice Crystals), American Institute of Aeronautics and Astronautics, 2013. doi:10.2514/6.2013-2674

[27] M. Burns, A Selection of Experimental Test Cases for the Validation of CFD Codes, Tech. rep., AGARD AR 303, Vol. I, chapter 5 (1994).

[28] P. R. Spalart, S. R. Allmaras, A one-equation turbulence model for aerodynamic flows, AIAA Paper 920439, 1992.

\section{Acknowledgement}

The research leading to these results has received funding from the European Union Seventh Framework Programme FP7/2007-2013 under grant agreement $n^{\circ}$ ACP2GA-2012-314314 (HAIC - High Altitude Ice Crystals). The author would like to thank Christian Bartels, Airbus Group Hamburg for his support and fruitful discussions during the detailed implementation of the features described in this paper. 